\title{
Corticosteroid therapy in acute respiratory distress syndrome
}

\author{
François Lamontagne MD MSc, Roy Brower MD, Maureen Meade MD MSc
}

Competing interests:

François Lamontagne and

Maureen Meade have

received an award from the

Canadian Institutes for

Health Research for

reasearch on corticosteroids

and acute respiratory

distress syndrome. Roy

Brower is a member of the

Data and Safety Monitoring

Board for AstraZeneca. No

other competing interests

were declared.

This article has been peer reviewed.

Correspondence to:

François Lamontagne,

francois.lamontagne

@ usherbrooke.ca

CMAJ 2013. DOI:10.1503 /cmaj.120582
$\mathrm{B}$ ecause acute respiratory distress syndrome (Figure 1) affects up to 150000 patients and causes 60000 deaths each year in the United States, the interest in potential therapies for this syndrome is high. Despite many candidate pharmacological interventions such as ketoconazole, ${ }^{1}$ lisofylline ${ }^{2}$ and inhaled nitric oxide, ${ }^{3}$ no drug has conclusively been shown to reduce mortality associated with the condition. However, some experts believe that corticosteroids constitute an exception to this rule, and have recommended corticosteroid therapy for severe cases of early $(<14 \mathrm{~d})$ disease. $^{4}$

Although clinical practice recommendations provide a point of reference for clinicians, their impact is often limited by the lack of strong underlying evidence. Irregular use of corticosteroids by physicians who care for patients with this syndrome suggests there is a lack of certainty regarding their effects. This review focuses on the use of systemic corticosteroids to treat acute respiratory distress syndrome, and attempts to bring clinical context to current evidence. A summary of the evidence used in this review is found in Box 1. The review is based primarily on randomized controlled trials and meta-analyses (effects of corticosteroids), and observational studies (predicting responsiveness).

\section{What is the underlying rationale for corticosteroid therapy?}

In the early 1980s, clinical investigators found that the inflammatory exudate from patients with adult respiratory distress syndromes could be reduced with high doses of systemic corticosteroids. ${ }^{5}$ More recently, in animal models of acute lung injury, Rocco and colleagues showed that corticosteroids reduce lung elastance and the deposition of collagen fibres in the extracellular matrix of the lung. ${ }^{6}$ Meduri and colleagues have seen that peripheral blood leukocytes exposed to plasma from patients with acute respiratory distress syndrome produce inflammatory cytokines, and that giving methylprednisolone to these patients reduced this production. ${ }^{7}$ Meduri and colleagues also found that procollagen aminoterminal propeptides (types I and III) in plasma and bronchoalveolar lavage increased in patients with nonresolving acute respiratory distress syndrome, and that the use of corticosteroids was associated with reductions in the levels of these markers. Moreover, the authors reported a substantial correlation between reductions in biomarker levels and improvements in lung injury scores. ${ }^{8}$ These preclinical and clinical investigations are among the many supporting a therapeutic antiinflammatory effect of corticosteroid therapy in acute respiratory distress syndrome in adults.

\section{Should corticosteroids be given to every patient with this syndrome?}

Current systematic reviews addressing the role of corticosteroid therapy in acute respiratory distress syndrome have not conclusively shown a survival benefit, even among specific subgroups. Six randomized clinical trials (RCTs) specifically examined the effects of corticosteroids on mortality associated with acute lung injury or acute respiratory distress syndrome between 1985 and 2007.9-14 The number of patients involved in these studies ranged from 16 to $180 .^{10,13}$ Mortality was measured at 45 days, at 180 days or after discharge from hospital. In early studies by Bernard and colleagues, ${ }^{9}$ Laggner and colleagues, ${ }^{10}$ and Weigelt and colleagues,${ }^{14}$ systemic corticosteroids had no impact on mortality; however, consistent with preliminary data showing reduced alveolar permeability with pharmacologic doses of corticosteroids, the tested therapies were short (limited to 1 or $2 \mathrm{~d})$ and the doses were high $(120 \mathrm{mg} / \mathrm{kg}$ 
methylprednisolone daily in 2 studies $^{9,14}$ and $8 \mathrm{~g} / \mathrm{d}$ prednisolone in 1 study $^{10}$ ). In 1998, Meduri and colleagues $^{12}$ published an RCT involving 24 patients, 16 of whom received a 32-day course of methylprednisolone ( $2 \mathrm{mg} / \mathrm{kg}$ daily), and 8 of whom were given a matching placebo. Mortality in hospital was lower for the group receiving corticosteroids $(62.5 \%$ for placebo v. $12.5 \%$ for corticosteroids; relative risk [RR] 0.2, $p=0.04) .{ }^{12}$ This study uniquely focused on patients who had unresolving acute respiratory distress syndrome after 7 days of mechanical ventilation, and is one of the reasons systemic corticosteroids subsequently found a niche in the treatment of "late-phase acute respiratory distress syndrome."

In an attempt to replicate these findings in a large, multicentre trial, the Acute Respiratory Distress Syndrome Network conducted the Late Steroid Rescue Study (LaSRS) ${ }^{13}$ in which patients received a 25-day course of methylprednisolone ( $2 \mathrm{mg} / \mathrm{kg}$ daily) or a matching placebo after at least 7 days of mechanical ventilation. Although similar to the Meduri study in many ways, LaSRS differed in that corticosteroids were discontinued, rather than tapered, 3 days after extubation, potentially preventing recrudescence of respiratory failure. Moreover, the LaSRS protocol did not involve active screening for infection. Mortality at 60 days was similar between the 2 groups $(28.6 \%$ for placebo v. $29.2 \%$ for corticosteroids; RR 1.0, $p=1.0$ ). In an a priori defined subgroup analysis, however, the duration of mechanical ventilation before the start of treatment with the study drug appeared to influence the effect of treatment. For the subgroup of patients given corticosteroids between 14 and 28 days after the start of mechanical ventilation, mortality was significantly higher compared with placebo ( $8 \%$ for placebo v. $35 \%$ for corticosteroids; RR 4.35, $p=0.02$ ). When corticosteroids were started within 7-13 days of mechanical ventilation, no significant difference in mortality was seen (36\% for placebo v. $27 \%$ for corticosteroids; RR $0.75, p=0.26$ ).

The most recent trial of corticosteroids for acute respiratory distress syndrome focused on early therapy (patients could be enrolled up to $72 \mathrm{~h}$ after meeting the criteria for a diagnosis of acute respiratory distress syndrome) and used a more modest dose of corticosteroids than in the previous trials $(1 \mathrm{mg} / \mathrm{kg}$ daily for $28 \mathrm{~d}) .{ }^{11} \mathrm{En}$ rolling 91 patients ( 28 in the placebo arm, 63 in the corticosteroid arm), this study failed to detect a statistically significant benefit in terms of mortality in hospital (42.9\% for placebo v. $23.8 \%$ for corticosteroids; RR 0.56, $p=0.07$ ).

Just as clinical trials addressing this issue have varied both in their design and their results, systematic reviews vary in their selection of studies for inclusion and in their results. Only 1 metaanalysis reported that corticosteroids reduce mortality in acute respiratory distress syndrome. ${ }^{15}$ This conclusion hinged on the inclusion of a small number of available studies selected on the basis of illness-defining terms in use since the mid 1990s, and 3 of the studies might have been biased by stopping early for perceived benefit. Other metaanalyses have been inconclusive, unable to rule out the possibility of either benefit or harm with respect to patient mortality (Table 1). ${ }^{15-19}$ Overall, meta-analyses have mostly shown that, even after pooling, existing studies lack power to measure meaningful effects of corticosteroids.

In light of the variation in study populations and corticosteroid regimens across trials, it is possible that steroids are harmful in some situations and helpful in others. Treatments of low dose and long duration appear most promising, ${ }^{20}$ but determining whether corticosteroids truly reduce mortality associated with acute respiratory distress syndrome will require additional data. Meta-analyses of patient-level data offer the

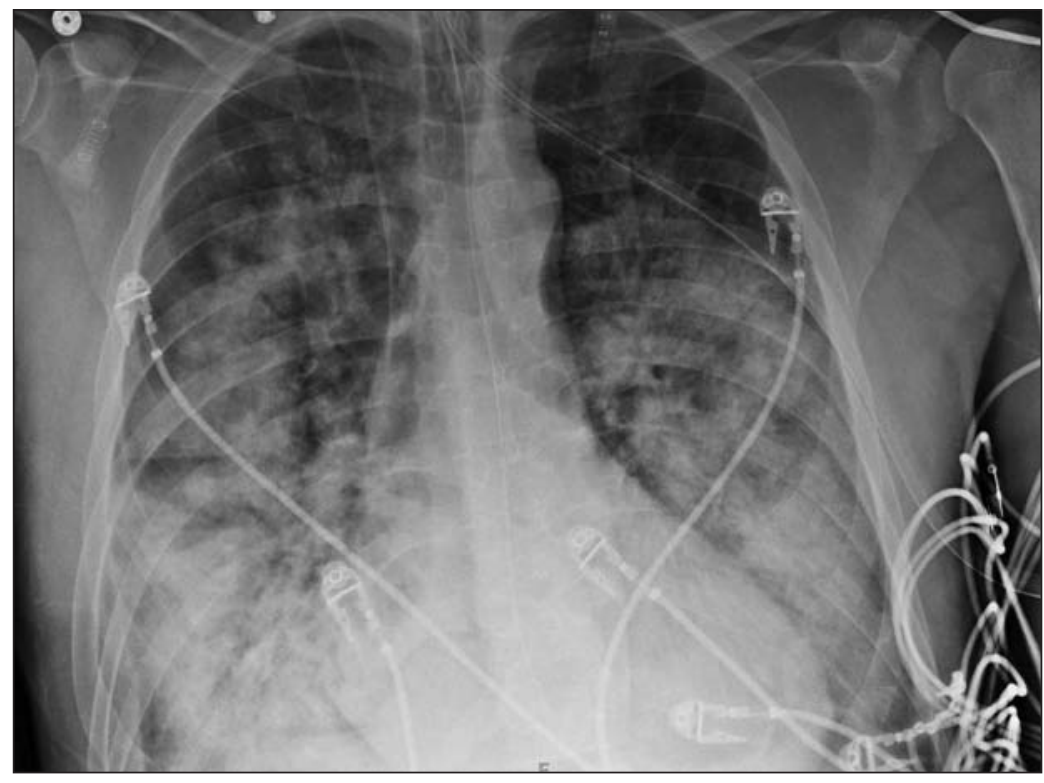

Figure 1: Chest radiograph showing opacifications in both lung fields, compatible with acute respiratory distress syndrome.

\section{Box 1: Evidence used in this review}

- We conducted a literature search of MEDLINE and Embase for the following terms appearing in the article text: "acute lung injury," "shock lung," "acute respiratory distress," "adult respiratory distress," "acute respiratory distress syndrome," "pneumonia," "glucocorticoid," "methylprednisolone," and "hydrocortisone." In addition, we searched for the following medical subject headings: "adult respiratory distress syndrome," "pneumonia," "adrenal cortex hormones," "steroids" and "glucocorticoids." We made no language-based restrictions to our literature search.

- To address each question, we identified the highest level of evidence from randomized controlled trials and meta-analyses, when available, or from observational studies. 
advantages of powerful subgroup analyses, which could resolve the problem of heterogeneous populations. Unfortunately, it is likely that even an individual patient meta-analysis would require more data than are currently available in studies of this syndrome. However, trials of corticosteroid therapy in septic shock, either already completed or currently underway, may provide sufficient data for this purpose if subgroups with acute respiratory distress syndrome are clearly delineated. In the meantime, corticosteroid therapy cannot be recommended for the routine management of acute respiratory distress syndrome.

\section{Is corticosteroid therapy an option for specific subsets of patients?}

As long as the effect of corticosteroid therapy on mortality remains uncertain, other potential benefits may have greater influence on a clinical decision to give or withhold corticosteroids. The most compelling additional outcome for which current research suggests potential benefit relates to the duration of mechanical ventilation. In a meta-analysis of the subset of studies that reported this outcome, corticosteroids were associated with 4.05 more ventilator-free days $(95 \%$ confidence interval [CI] 0.22-8.71). ${ }^{16}$

Moreover, the broad criteria defining acute res- piratory distress syndrome are similar to those of other illnesses that could respond to steroid therapy. These may simply accompany the syndrome or constitute the underlying cause of the syndrome. Thus, despite the lack of evidence to suggest improved survival for the general population of patients with acute respiratory distress syndrome, there are some situations in which careful consideration of corticosteroid therapy is prudent. In the setting of diagnostic uncertainty, clinicians must consider the response to steroids of illnesses that can masquerade as acute respiratory distress syndrome (i.e., profound hypoxemia and diffuse bilateral opacification on chest radiography). Examples of such conditions include cryptogenic organizing pneumonia and acute eosinophilic pneumonia, for which corticosteroids are a mainstay of therapy, and acute interstitial pneumonia, for which the role of corticosteroids remains uncertain. Pneumonia caused by specific infectious agents such as Pneumocystis jiroveci and tuberculosis are additional steroid-responsive illnesses to consider. ${ }^{21}$ Although these illnesses are uncommon, in the absence of a clear risk factor for acute respiratory distress syndrome, their likelihood increases, and the potential for steroid responsiveness is an important consideration.

Other illnesses more commonly seen in the intensive care unit, such as severe bronchoconstriction and vasopressor-refractory septic shock,

Table 1: Reviews on corticosteroids in acute lung injury and acute respiratory distress syndrome

\begin{tabular}{|c|c|c|c|c|c|}
\hline Review & $\begin{array}{l}\text { Total no. of studies } \\
\text { (no. of included RCTs) }\end{array}$ & $\begin{array}{l}\text { Populations of interest } \\
\text { in primary studies }\end{array}$ & $\begin{array}{l}\text { Primary } \\
\text { outcome }\end{array}$ & Results & $\begin{array}{c}\text { Comments on subgroup } \\
\text { analyses }\end{array}$ \\
\hline $\begin{array}{l}\text { Meduri et al. } \\
2008^{15}\end{array}$ & $5 \quad(5)$ & $\begin{array}{l}\text { Acute lung injury, } \\
\text { acute respiratory } \\
\text { distress syndrome }\end{array}$ & Mortality & $\begin{array}{c}\text { RR } 0.76 \\
(0.62-0.93)\end{array}$ & $\begin{array}{l}\text { Size of primary studies, } \\
\text { no interaction test }\end{array}$ \\
\hline $\begin{array}{l}\text { Peter et al. } \\
2008^{16}\end{array}$ & $\begin{array}{c}5(5) \\
\text { addressed } \\
\text { therapeutic use }\end{array}$ & $\begin{array}{l}\text { Acute respiratory } \\
\text { distress syndrome or } \\
\text { patients at risk of } \\
\text { syndrome }\end{array}$ & Mortality & $\begin{array}{l}\text { OR } 0.62 \\
(0.23-1.26)\end{array}$ & $\begin{array}{l}\text { Timing, dose and year of } \\
\text { study completion not } \\
\text { significant }\end{array}$ \\
\hline $\begin{array}{l}\text { Lamontagne } \\
\text { et al. } 2009^{17}\end{array}$ & $12(12)$ & $\begin{array}{l}\text { Acute lung injury, } \\
\text { acute respiratory } \\
\text { distress syndrome, } \\
\text { severe pneumonia }\end{array}$ & Mortality & RR $0.84(0.66-1.06)$ & $\begin{array}{l}9 \text { trials of low-dose } \\
\text { corticosteroids RR } 0.68 \\
(0.49-0.96) ; p=0.047\end{array}$ \\
\hline $\begin{array}{l}\text { Tang et al. } \\
2009^{18}\end{array}$ & $9 \quad(4)$ & $\begin{array}{l}\text { Acute lung injury, } \\
\text { acute respiratory } \\
\text { distress syndrome }\end{array}$ & Mortality & $\begin{array}{l}\text { RCTs RR } 0.51 \\
(0.24-1.09)\end{array}$ & $\begin{array}{l}\text { Timing, tapering, drug } \\
\text { formulation, year of } \\
\text { study completion, } \\
\text { crossover design not } \\
\text { significant }\end{array}$ \\
\hline \multirow[t]{2}{*}{$\begin{array}{l}\text { Agarwal et al. } \\
2007^{19}\end{array}$} & \multirow[t]{2}{*}{$6(4)$} & \multirow[t]{2}{*}{$\begin{array}{l}\text { Acute lung injury, } \\
\text { acute respiratory } \\
\text { distress syndrome }\end{array}$} & \multirow[t]{2}{*}{ Mortality } & $\begin{array}{l}\text { Early acute respiratory } \\
\text { distress synrome } \\
\text { OR } 0.57(0.25-1.32)\end{array}$ & \multirow[t]{2}{*}{ Not available } \\
\hline & & & & $\begin{array}{l}\text { Late acute respiratory } \\
\text { distress syndrome } \\
\text { OR } 0.58(0.22-1.53)^{*}\end{array}$ & \\
\hline
\end{tabular}


may also be reasonable indications for the use of corticosteroids. Co-occurrence of steroidresponsive illnesses and acute respiratory distress syndrome renders trials even more difficult to interpret. Future research should be powered to distinguish the effects of corticosteroids on acute respiratory distress syndrome from their effects on copresenting illnesses.

\section{What are the potential harms of corticosteroid therapy?}

Physicians have been prescribing corticosteroids for decades and have learned to recognize and anticipate many of their harmful effects, such as severe secondary infections, stress ulceration, poor wound healing, psychoses and hyperglycemia. ${ }^{22}$ Most of these complications can have severe consequences for patients with critical illness. Because these patients are at risk of a variety of serious complications related to their acute or chronic illnesses, it is often challenging to establish causality between therapies and potential adverse effects outside of clinical trials. ${ }^{23}$

To date, the harms of corticosteroids have received insufficient attention in critical care clinical trials. ${ }^{23}$ A systematic review of corticosteroid therapy for acute respiratory distress syndrome found that high-dose regimens were associated with increased risk of infection (RR 1.77, 95\% CI 1.23-2.54). ${ }^{17}$ In contrast, data from LaSRS suggest no increase in severe infections with a lowdose regimen. ${ }^{13}$ Unfortunately, definitions and screening protocols for infections vary considerably between studies, ${ }^{16}$ and most did not adequately define, systematically screen for or adjudicate infectious complications. ${ }^{17}$ Exceptions are 2 clinical trials by Meduri and colleagues, which involved vigilant protocols to screen for new infections; however, with a total of 24 and 91 patients, the studies were underpowered to detect differences in rates of infection. ${ }^{11,12}$

The association between corticosteroid therapy and weakness acquired during intensive care remains uncertain. An important concern that corticosteroids may affect the nerves and muscles of patients with critical illness comes from the largest trial of corticosteroids for patients with acute respiratory distress syndrome, in which neuromyopathy was reported in 9 patients, all of whom were in the corticosteroid arm $(p=0.001){ }^{13}$ Although the number of events in this study are too few to form firm conclusions, the results are supported by a recent case-control study that reported a statistically significant association between corticosteroid therapy and neuromuscular disability in patients in intensive care. ${ }^{24}$
Overall, research programs focusing on the efficacy of corticosteroid therapy in acute respiratory distress syndrome have insufficiently addressed the harm it causes in this highly vulnerable population. Designs of future trials of corticosteroids in acute respiratory distress syndrome will have to incorporate rigorous measures of clinically relevant new infections, weakness acquired during intensive care, gastrointestinal bleeding, and metabolic and psychiatric disorders. Equally important will be the implementation of systematic screening or independent adjudication based on a priori defined criteria for each adverse event. In an analogous situation, a large multicentre trial of steroid therapy for traumatic brain injury was stopped early after the investigators measured excess death and severe disability in the treatment arm. ${ }^{25,26}$ Before that study, statistical pooling of the results from 16 smaller studies had not shown excess death or harm with highdose corticosteroids. ${ }^{26}$

\section{Can we predict responsiveness to corticosteroid therapy?}

In an ideal world, clinicians could access a test for steroid responsiveness in the heterogeneous population of patients with acute respiratory distress syndrome. Several assays of corticosteroid sensitivity are currently available for clinical research purposes. ${ }^{27-34}$ One ex vivo assay measures suppression by dexamethasone of phytohemagglutinininduced proliferation of lymphocytes in peripheral blood. Other ex vivo assays measure dexamethasone suppression of lipopolysaccharide-induced production of pro-inflammatory cytokines, such as interleukin- 6 and tumour necrosis factor $\alpha$, and stimulation of anti-inflammatory proteins, such as the glucocorticoid-induced leucine zipper and FKBP51, from mononuclear cells in peripheral blood. ${ }^{3436}$

Two important observations are apparent in previous studies of corticosteroid responsiveness. First, there is great variability between peoples' sensitivities to corticosteroids. ${ }^{27-31,33,35,37-39}$ For example, the normal range of dexamethasoneinduced inhibition of lymphocyte proliferation (after stimulation with phytohemagglutinin) is $10 \%-98 \% .^{28}$ There are several reasons for such highly variable responses. Some polymorphisms of the gene encoding the glucocorticoid receptor can diminish or enhance sensitivity to glucocorticoids, ${ }^{40,41}$ but these polymorphisms are uncommon and unlikely to explain such wide variations in sensitivity. Most differences in corticosteroid sensitivity between people are related to variations in transcription of the glucocorticoid recep- 
tor gene and to posttranscriptional and posttranslational modifications. These differences depend on several factors, including specific tissue and inflammatory milieu. ${ }^{42-45}$ The range of variation in corticosteroid responsiveness among people suggests that, in previous trials, corticosteroids may have had beneficial effects in some patients, but not beneficial (or even harmful) effects in others.

Second, some assays predicted clinical response to corticosteroids of inflammatory conditions such as ulcerative colitis, ${ }^{31}$ asthma, ${ }^{27,29,44,46,47}$ psoriasis $^{48}$ and rheumatoid arthritis. ${ }^{49}$ An assay of corticosteroid responsiveness may provide critical information to distinguish patients with acute respiratory distress syndrome who will respond well to corticosteroid therapy from those who will not.

\section{Gaps in knowledge}

Editorialists have emphasized the need for more conclusive data, ${ }^{50}$ but future studies on the efficacy of corticosteroid therapy for acute respiratory distress syndrome will face many challenges. Competing indications and contraindications for corticosteroid therapy among patients with this syndrome threaten their eligibility to participate in such studies and ongoing adherence to protocol by causing many patients in the control arm to also receive corticosteroids. The evolving literature on the role of corticosteroids in sepsis, in particular, will constitute a substantial challenge if patients who also have septic shock are not enrolled in trials.

Firmly held beliefs about the role of corticosteroids in acute respiratory distress syndrome and entrenched practice patterns may affect decision-making among some intensive care specialists, compromising the feasibility of future RCTs.

Other challenges include the need for complex, data-heavy, resource-intensive measurement of myriad potential adverse effects of corticosteroid therapy in the intensive care unit. The lack of evidence concerning the potential harm caused by corticosteroids represents the largest gap in previous studies, but rigorous measures of these outcomes are costly. In addition, complex and resource-intensive measurement of the multiple effects of corticosteroids is required, specifically those pertaining to the immune regulatory, metabolic, endocrine, vasoregulatory and neurocognitive functions. Validating ways of obtaining more reliable measurement will thus generate considerable costs.

There are essentially 3 potential paths forward. Investigators could consider a large pragmatic RCT comparing early, prolonged corticosteroid therapy to placebo, and providing liberal criteria for giving steroids in the placebo arm. This approach might be acceptable to most intensivists, but a prohibitively large sample may be required. Alternatively, investigators could target a distinct, as yet unspecified, subgroup of patients with acute respiratory distress syndrome who have no competing indications for corticosteroids, thus dealing with the problem of heterogeneous patient populations in previous studies. A disadvantage of this strategy is that it would impose new feasibility challenges and limit generalizability. A third option is to conduct a review of individual patient data, with meta-analysis including all trials of corticosteroid therapy in acute respiratory distress syndrome, in addition to trials of corticosteroids in sepsis, for which discreet subgroups of acute respiratory distress syndrome are clearly defined. An example of this option is the Adjunctive Corticosteroid Treatment in Critically Ill Patients With Septic Shock study (clinicaltrials.gov registry no. NCT01448109) currently underway in Australia and New Zealand, with a target enrolment of 3800 patients.

\section{Conclusion}

The role of corticosteroids in managing acute respiratory distress syndrome remains uncertain. Despite a strong physiologic rationale with supporting evidence from laboratory research, clinical trials have yet to establish that the therapy improves survival, and the threat of important and long-term complications looms large. Specific subgroups with a high likelihood of responding to treatment remain undefined, and bedside tests for responsiveness in this setting are not sufficiently developed for use in routine clinical care.

Although current evidence does not support routine use of corticosteroids to treat this syndrome, there are specific situations where their use is prudent; for example, when an alternative diagnosis or underlying cause will respond to treatment with steroids (e.g., cryptogenic organizing pneumonia). Special challenges to conducting definitive trials in this field have thwarted investigators in the past. For the near future, clinicians can look forward to the completion of related clinical trials.

\section{References}

1. Ketoconazole for early treatment of acute lung injury and acute respiratory distress syndrome: a randomized controlled trial. The ARDS Network. JAMA 2000;283:1995-2002.

2. Randomized, placebo-controlled trial of lisofylline for early treatment of acute lung injury and acute respiratory distress syndrome. Crit Care Med 2002;30:1-6.

3. Adhikari NK, Burns KE, Friedrich JO, et al. Effect of nitric oxide on oxygenation and mortality in acute lung injury: systematic review and meta-analysis. BMJ 2007;334:779.

4. Annane D. Glucocorticoids for ARDS: just do it! Chest 2007; 131:945-6.

5. Sibbald WJ, Anderson RR, Reid B, et al. Alveolo-capillary permeability in human septic ARDS. Effect of high-dose corticosteroid therapy. Chest 1981;79:133-42. 
6. Rocco PR, Souza AB, Faffe DS, et al. Effect of corticosteroid on lung parenchyma remodeling at an early phase of acute lung injury. Am J Respir Crit Care Med 2003;168:677-84.

7. Meduri GU, Tolley EA, Chrousos GP, et al. Prolonged methylprednisolone treatment suppresses systemic inflammation in patients with unresolving acute respiratory distress syndrome: evidence for inadequate endogenous glucocorticoid secretion and inflammation-induced immune cell resistance to glucocorticoids. Am J Respir Crit Care Med 2002;165:983-91.

8. Meduri GU, Tolley EA, Chinn A, et al. Procollagen types I and III aminoterminal propeptide levels during acute respiratory distress syndrome and in response to methylprednisolone treatment. Am J Respir Crit Care Med 1998;158:1432-41.

9. Bernard GR, Luce JM, Sprung CL, et al. High-dose corticosteroids in patients with the adult respiratory distress syndrome. N Engl J Med 1987;317:1565-70.

10. Laggner AN, Lenz K, Base W, et al. Effect of high-dose prednisolone on lung fluid in patients with non-cardiogenic lung edema [article in German]. Wien Klin Wochenschr 1987;99:245-9.

11. Meduri GU, Golden E, Freire AX, et al. Methylprednisolone infusion in early severe ARDS: results of a randomized controlled trial. Chest 2007;131:954-63.

12. Meduri GU, Headley AS, Golden E, et al. Effect of prolonged methylprednisolone therapy in unresolving acute respiratory distress syndrome: a randomized controlled trial. JAMA 1998;280: 159-65.

13. Steinberg KP, Hudson LD, Goodman RB, et al. Efficacy and safety of corticosteroids for persistent acute respiratory distress syndrome. N Engl J Med 2006;354:1671-84.

14. Weigelt JA, Norcross JF, Borman KR, et al. Early steroid therapy for respiratory failure. Arch Surg 1985;120:536-40.

15. Meduri GU, Marik PE, Chrousos GP et al. Steroid treatment in ARDS: a critical appraisal of the ARDS network trial and the recent literature. Intens Care Med 2008;34:61-9.

16. Peter JV, John P, Graham PL, et al. Corticosteroids in the prevention and treatment of acute respiratory distress syndrome (ARDS) in adults: meta-analysis. BMJ 2008;336:1006-9.

17. Lamontagne F, Briel M, Guyatt GH, et al. Corticosteroid therapy for acute lung injury, acute respiratory distress syndrome, and severe pneumonia: a meta-analysis of randomized controlled trials. J Crit Care 2010;25:420-35.

18. Tang BM, Craig JC, Eslick GD, et al. Use of corticosteroids in acute lung injury and acute respiratory distress syndrome: a systematic review and meta-analysis. Crit Care Med 2009;37:1594 603.

19. Agarwal R, Nath A, Aggarwal AN, et al. Do glucocorticoids decrease mortality in acute respiratory distress syndrome? A meta-analysis. Respirology 2007;12-585-90.

20. Meduri GU, Marik PE, Annane D. Prolonged glucocorticoid treatment in acute respiratory distress syndrome: Evidence supporting effectiveness and safety. Crit Care Med 2009;37:1800-3.

21. McGee S, Hirschmann J. Use of corticosteroids in treating infectious diseases. Arch Intern Med 2008;168:1034-46.

22. Fardet L, Kassar A, Cabane J, et al. Corticosteroid-induced adverse events in adults: frequency, screening and prevention. Drug Saf 2007;30:861-81.

23. Cook D, Lauzier F, Rocha MG, et al. Serious adverse events in academic critical care research. CMAJ 2008;178:1181-4

24. Rady MY, Johnson DJ, Patel B, et al. Corticosteroids influence the mortality and morbidity of acute critical illness. Crit Care 2006;10:R101.

25. Edwards P, Arango M, Balica L, et al. Final results of MRC CRASH, a randomised placebo-controlled trial of intravenous corticosteroid in adults with head injury-outcomes at 6 months. Lancet 2005;365:1957-9.

26. Roberts I, Yates D, Sandercock P, et al. Effect of intravenous corticosteroids on death within 14 days in 10008 adults with clinically significant head injury (MRC CRASH trial): randomised placebo-controlled trial. Lancet 2004;364:1321-8.

27. Brown PH, Teelucksingh S, Matusiewicz SP, et al. Cutaneous vasoconstrictor response to glucocorticoids in asthma. Lance 1991;337:576-80

28. Chriguer RS, Elias LL, da Silva IM Jr, et al. Glucocorticoid sensitivity in young healthy individuals: in vitro and in vivo studies. $J$ Clin Endocrinol Metab 2005;90:5978-84.

29. Corrigan CJ, Brown PH, Barnes NC, et al. Glucocorticoid resistance in chronic asthma. Glucocorticoid pharmacokinetics, glucocorticoid receptor characteristics, and inhibition of peripheral blood $\mathrm{T}$ cell proliferation by glucocorticoids in vitro. Am Rev Respir Dis 1991;144:1016-25.

30. DeRijk RH, Eskandari F, Sternberg EM. Corticosteroid resistance in a subpopulation of multiple sclerosis patients as measured by ex vivo dexamethasone inhibition of LPS induced IL-6 production. J Neuroimmunol 2004;151:180-8.
31. Hearing SD, Norman M, Probert CS, et al. Predicting therapeutic outcome in severe ulcerative colitis by measuring in vitro steroid sensitivity of proliferating peripheral blood lymphocytes. Gut 1999;45:382-8.

32. Place VA, Velazquez JG, Burdick KH. Precise evaluation of topically applied corticosteroid potency. Modification of the Stoughton-McKenzie assay. Arch Dermatol 1970;101:531-7.

33. Smit P, Russcher H, de Jong FH, et al. Differential regulation of synthetic glucocorticoids on gene expression levels of glucocorticoid-induced leucine zipper and interleukin-2. J Clin Endocrinol Metab 2005;90:2994-3000.

34. Vermeer H, Hendriks-Stegeman BI, van der Burg B, et al. Glucocorticoid-induced increase in lymphocytic FKBP51 messenger ribonucleic acid expression: a potential marker for glucocorticoid sensitivity, potency, and bioavailability. J Clin Endocrinol Metab 2003;88:277-84.

35. Vermeer H, Hendriks-Stegeman BI, van Suylekom D, et al. An in vitro bioassay to determine individual sensitivity to glucocorticoids: induction of FKBP51 mRNA in peripheral blood mononuclear cells. Mol Cell Endocrinol 2004;218:49-55.

36. Vermeer H, Hendriks-Stegeman BI, Verrijn Stuart AA, et al. A comparison of in vitro bioassays to determine cellular glucocorticoid sensitivity. Eur J Endocrinol 2004;150:41-7.

37. Hearing SD, Norman M, Smyth $\mathrm{C}$, et al. Wide variation in lymphocyte steroid sensitivity among healthy human volunteers. J Clin Endocrinol Metab 1999;84:4149-54.

38. Molijn GJ, Spek JJ, van Uffelen JC, et al. Differential adaptation of glucocorticoid sensitivity of peripheral blood mononuclear leukocytes in patients with sepsis or septic shock. J Clin Endocrinol Metab 1995;80:1799-803.

39. Schleimer RP, Schulman ES, MacGlashan DW Jr., et al. Effects of dexamethasone on mediator release from human lung fragments and purified human lung mast cells. J Clin Invest 1983; 71:1830-5.

40. Hon YY, Jusko WJ, Spratlin VE, et al. Altered methylprednisolone pharmacodynamics in healthy subjects with histamine $N$-methyltransferase C314T genetic polymorphism. J Clin Pharmacol 2006;46:408-17.

41. Manenschijn L, van den Akker EL, Lamberts SW, et al. Clinical features associated with glucocorticoid receptor polymorphisms. An overview. Ann N Y Acad Sci 2009;1179:179-98.

42. Meduri GU, Yates CR. Systemic inflammation-associated glucocorticoid resistance and outcome of ARDS. Ann N Y Acad Sci 2004;1024:24-53.

43. Revollo JR, Cidlowski JA. Mechanisms generating diversity in glucocorticoid receptor signaling. Ann N Y Acad Sci 2009;1179: 167-78

44. Rhen T, Cidlowski JA. Antiinflammatory action of glucocorticoids-new mechanisms for old drugs. N Engl J Med 2005;353: 1711-23

45. Zhou J, Cidlowski JA. The human glucocorticoid receptor: one gene, multiple proteins and diverse responses. Steroids 2005;70: 407-17.

46. Alvarez J, Surs W, Leung DY, et al. Steroid-resistant asthma: immunologic and pharmacologic features. J Allergy Clin Immunol 1992;89:714-21.

47. Bhavsar P, Hew M, Khorasani N, et al. Relative corticosteroid insensitivity of alveolar macrophages in severe asthma compared with non-severe asthma. Thorax 2008;63:784-90.

48. Cornell RC, Stoughton RB. Correlation of the vasoconstriction assay and clinical activity in psoriasis. Arch Dermatol 1985;121: 63-7.

49. Kirkham BW, Corkill MM, Davison SC, et al. Response to glucocorticoid treatment in rheumatoid arthritis: in vitro cell mediated immune assay predicts in vivo responses. J Rheumatol 1991;18:821-5.

50. Thompson BT. Corticosteroids for ARDS. Minerva Anestesiol 2010;76:441-7.

Affiliations: From the Centre de Recherche Clinique ÉtienneLe Bel and Department of Internal Medicine (Lamontagne), University of Sherbrooke, Sherbrooke, Que.; Pulmonary and Critical Care Medicine (Brower), Johns Hopkins University, Baltimore, Md.; and Hamilton Health Sciences, Departments of Medicine and Clinical Epidemiology and Biostatistics (Meade), McMaster University, Hamilton, Ont.

Contributors: All of the authors contributed to the conception and design of this review, the acquisition and interpretation of the data and the writing of the manuscript. All of the authors approved the final version submitted for publication. 